\title{
The Radiation Sensitivity of Normal and Polyoma-Transformed Rat Embryo Cells ${ }^{1}$
}

\author{
J. F. WILLIAMS ${ }^{2}$ AND J. E. TILL \\ Department of Medical Biophysics, University of Toronto, and The Ontario Cancer Institute, \\ Toronto, Canada
}

\section{INTRODUCTION}

Many determinations have been made of the radiosensitivity of the proliferative capacity of mammalian cells. These have been carried out on several different cell lines, both normal and malignant, tested by a variety of techniques, in a number of different laboratories. The results of these determinations (for a summary of data, see ref. 1) have led to the view that normal and malignant mammalian cells derived from different sources differ relatively little in their responses to ionizing radiations, and that the radiosensitivity of cells tested in vitro is similar to that of cells tested in vivo (2-5). Although the available evidence provides strong support for these views, they have not been tested directly with the same cell system, mainly because of the lack of a system suitable for such a test. The finding $(6,7)$ that rodent embryo cells infected in vitro with polyoma virus may rapidly undergo a transformation in cell properties, and that these "transformed" cells are usually also malignant, in that they are capable of forming tumors when transplanted into suitable adult animals, provided an approach to this problem. Although transformed cells may be obtained from polyoma-infected cultures of either mouse, hamster, or rat embryo cells, the rat embryo cell system appears to be most suitable for radiobiological studies, since quantitative techniques are available for measuring colony formation by both normal and transformed cells in vitro (8), and by malignant cells in vivo $(9,10)$. Using this system, we have tested for the effect of polyoma virus infection leading to transformation on the radiosensitivity of the colony-forming ability of rat embryo cells. Also, the radiosensitivity of cells, based on the survival of their ability to form colonies in vitro, has been compared vith that of the same type of cells tested in vivo. The results of these studies are described in this report.

1 This paper is based on a thesis submitted by J. F. Williams in conformity with the requirements for the degree of Doctor of Philosophy in the University of Toronto.

${ }^{2}$ Present address: Virus Laboratory, University of California, Berkeley, California. 


\section{MATERIALS AND METHODS}

Cells

Normal rat embryo cell cultures were prepared from 16- to 18-day embryos of the Buffalo inbred strain (obtained from Simonsen Labs., Gilroy, California) as described elsewhere (8). Uncloned secondary cultures were used as a source of normal cells.

Transformed cell lines were obtained by the methods described previously $(8,9)$. Prior to infection, primary rat embryo cell cultures were dispersed by treatment with $0.1 \%$ trypsin solution in phosphate-buffered saline (PBS) (11), and $2 \times 10^{6}$ cells were seeded onto each of a number of $60-\mathrm{mm}$ plastic petri dishes (Falcon Plastics, Los Angeles) in growth medium. Growth medium was CMRL 1066 (12) plus $10 \%$ calf serum (Flow Laboratories, Rockville, Maryland). After 48 hours of incubation at $37^{\circ} \mathrm{C}$ in a humidity- and $\mathrm{CO}_{2}$-controlled incubator, the cells were washed with PBS and infected with polyoma virus (strain TSP1; 13) at a multiplicity of 1000 plaque-forming units per cell. After 2 hours for adsorption, the cells were washed free of virus, the cultures were dispersed with trypsin, and an appropriate cell number was seeded onto plastic petri dishes containing $10^{5}$ irradiated (7000 rads) normal feeder cells in assay medium, which consisted of CMRL 1066 plus $5 \%$ by volume of a selected batch of calf serum (obtained from Woodlyn Farms, Guelph, Ontario). Selection of this particular batch of serum for use in the assay medium was based on the observation that the characteristic morphology of transformed colonies was more easily recognized in the presence of a monolayer of cells of normal morphology ( 8 ) when the cells were grown in medium containing serum from this particular batch. After 12 days of incubation at $37^{\circ} \mathrm{C}$, distinct, isolated transformed foci of cells were picked from the infected cultures by excision with a Pasteur pipet, seeded onto dishes containing feeder cells, and again cultured as described above. Thereafter, the cells were subcultured, after appropriated dilutions, at approximately weekly intervals. The cell lines established in this way were termed transformed focal lines. "Long-term" transformed focal lines were lines which had been carried in cell culture for at least thirty-five weekly passages prior to use in the experiments reported here. These long-term focal lines were designated by the prefix f-for example, f12. "Short-term" transformed cell lines (E2, E3, and E4) were focal lines which had been in culture for not more than 6 to 7 weeks at the time the experiments were performed.

Newly transformed cells were obtained by two different modifications of the method described above for the isolation of transformed focal lines. In the first modification, the polyoma-infected cultures were washed free of virus, the growth medium was replaced, and the cultures were incubated for a further 36 hours before being dispersed for irradiation. In the second modification, the infected cultures were dispersed immediately after infection, reseeded in assay medium at a concentration of $5 \times 10^{5}$ cells per petri dish, and incubated for a period of 7 days. After 
this time, they were dispersed for irradiation. These cultures contained a mixture of transformed and nontransformed cells. With the exception of the newly transformed cells, all the cells tested were in the exponential growth phase at the time of irradiation.

\section{Irradiation Procedures}

Immediately prior to irradiation, the cells were dispersed by treatment with $0.1 \%$ trypsin solution, washed in PBS, and suspended in CMRL 1066 medium at a concentration of either $4 \times 10^{6}$ cells $/ \mathrm{ml}$ or $4 \times 10^{5}$ cells $/ \mathrm{ml}$. The cell suspensions were cooled to $4^{\circ} \mathrm{C}$ and irradiated in $12-\mathrm{ml}$ polyethylene dilution tubes (Falcon Plastics, Los Angeles) held perpendicular to the radiation beam in a plastic tube holder. Irradiations were carried out in a dual-source $\mathrm{Cs}^{137}$ irradiator (14) at a dose rate of $112 \mathrm{rads} / \mathrm{min}$ as determined with the ferrous ammonium sulfate dosimeter (15). During the irradiations, the cells were shaken when individual tubes were removed sequentially, and little or no clumping of the cells occurred. The time required for removal of each tube prior to continuing the irradiation was approximately $1 \mathrm{~min}$ ute. After irradiation, the cells were diluted immediately in CMRL 1066 and tested for survival of colony-forming ability.

Cells of the transformed lines were irradiated at $4 \times 10^{5}$ cells $/ \mathrm{ml}$. Survival of the f12 long-term focal line was tested in vivo by means of the lung colony assay, as well as in vitro by means of the colony assay. For this reason, since higher concentrations of cells were required, the 12 line was also irradiated at $4 \times 10^{6}$ cells $/ \mathrm{ml}$. When irradiated at these two cell concentrations and tested for survival of colonyforming ability in vitro, the f12 line was found to have the same radiosensitivity, which indicates that the use of these two different cell concentrations did not influence the results obtained. Further, no change in sensitivity of the f12 line was seen when $\mathrm{O}_{2}$ was bubbled through the cell suspension $\left(4 \times 10^{5}\right.$ cells $\left./ \mathrm{ml}\right)$ immediately before irradiation. These preliminary findings indicated that oxygen depletion had no influence on the results obtained for cells irradiated at the higher cell concentrations. Cells in newly infected cultures were irradiated at a concentration of $4 \times 10^{6}$ cells/ml, since large numbers of cells were required in vitro in the focus assay (see below) and in vivo in the lung assay. In the present experiments, normal rat embryo cells were also irradiated at $4 \times 10^{6}$ cells $/ \mathrm{ml}$.

\section{Determination of Colony-Forming Ability in Vitro}

After irradiation of transformed or normal cells, an appropriate number of cells was seeded onto $60-\mathrm{mm}$ plastic petri dishes containing $10^{5}$ heavily irradiated rat embryo feeder cells in growth medium (16). The cultures were incubated at $37^{\circ} \mathrm{C}$ for 7 to 9 days, after which time they were stained with methylene blue ( $1 \mathrm{gm} / \mathrm{liter})$ and the number of colonies was determined.

In experiments utilizing freshly infected cells, two different assay procedures 
were used. In the first, discrete colonies were obtained as described above, and differential counts of colonies with normal and transformed morphologies were made (17). In the second assay procedure, the cells were seeded, with $10^{5}$ feeder cells, in assay medium (see above). After 10 to 12 days of incubation, the cultures were stained with methylene blue, and foci of transformed cells were scored, as described elsewhere (8).

In assessing the survival of the most heavily irradiated samples by means of the focus assay, $8 \times 10^{5}$ cells were seeded to each dish. Since this was greater than the number of irradiated feeder cells per dish $\left(10^{5}\right)$ usually used in the focus assay, a test was made of the effect of increasing the number of feeder cells from $10^{5}$ to $8 \times 10^{5}$ cells per dish, on the number of foci formed. It was found that the reduction in the efficiency of focus formation due to the presence of the increased number of irradiated feeder cells was no more than $10 \%$ and thus was of negligible importance in these experiments.

\section{Determination of Colony-Forming Ability in Vivo}

After irradiation, suitable numbers of cells in $0.5 \mathrm{ml}$ of CMRL 1066 medium were injected intravenously into the lateral tail veins of weanling rats of the Buffalo inbred strain (obtained from Microbiological Associates, Bethesda, Maryland). Prior to injection of the cells, the animals were irradiated with 350 rads of total-body irradiation, from the same $\mathrm{Cs}^{137}$ dual-source irradiator used for irradiation of the cell suspensions. This dose of radiation was given in order to minimize the effects of any histocompatability differences which might have existed between the recipient animals and the transplanted cells (10). On the eighteenth day after injection of cells, the lungs were removed from the recipient animals and fixed in Bouin's solution. Counts of the number of colonies seen on the entire surface of each lobe of the lungs were made under twofold magnification. This assay procedure has been described in detail elsewhere $(9,10)$.

\section{Survival Curves}

Survival curves, showing the proportion of cells retaining colony-forming ability after various doses of irradiation, were obtained for each cell line. These survival curves, with one exception (the in vivo curve of Fig. 4), were measured to levels of survival of at least $10^{-3}$ of the control value. The survival curves obtained were of the usual type $\mathrm{C}$ form (see Fig. 1) and were characterized by two parameters $-D_{0}$, the dose required to reduce the surviving fraction by $63 \%$ on the exponential portion of the curve, and $n$, the extrapolation number, obtained by extrapolating the exponential portion of the curve back to zero dose on the semilogarithmic plot and reading off the fractional survival (18). The exponential portions of the curves were fitted to the experimental points by the method of least squares. 


\section{Growth Rates of Cells in Vitro}

The growth rates of cells in vitro were determined as follows: Replicate cultures were prepared by adding a known number of cells to $60-\mathrm{mm}$ plastic petri dishes containing growth medium. At intervals thereafter, two replicate cultures were taken, the cells were suspended by trypsin treatment, and the cell concentration in each suspension was determined from hemocytometer counts.

\section{Chromosome Numbers}

The ploidy of the cells of the various lines used was determined by counting the numbers of chromosomes present in cells prepared according to the method of Fox and Zeiss (19). Scoring of the preparations was limited to estimation of the proportion of near-diploid and near-tetraploid cells in the cell populations. Karyotypes for the different lines were not accurately characterized.

\section{RESULTS}

Radiosensitivity of Normal Rat Embryo Cells and of Cells of a Number of Long-Term Transformed Lines

To test for possible differences in radiosensitivity between normal rat embryo cells and transformed cells, the radiation survival curves for normal (freshly iso-

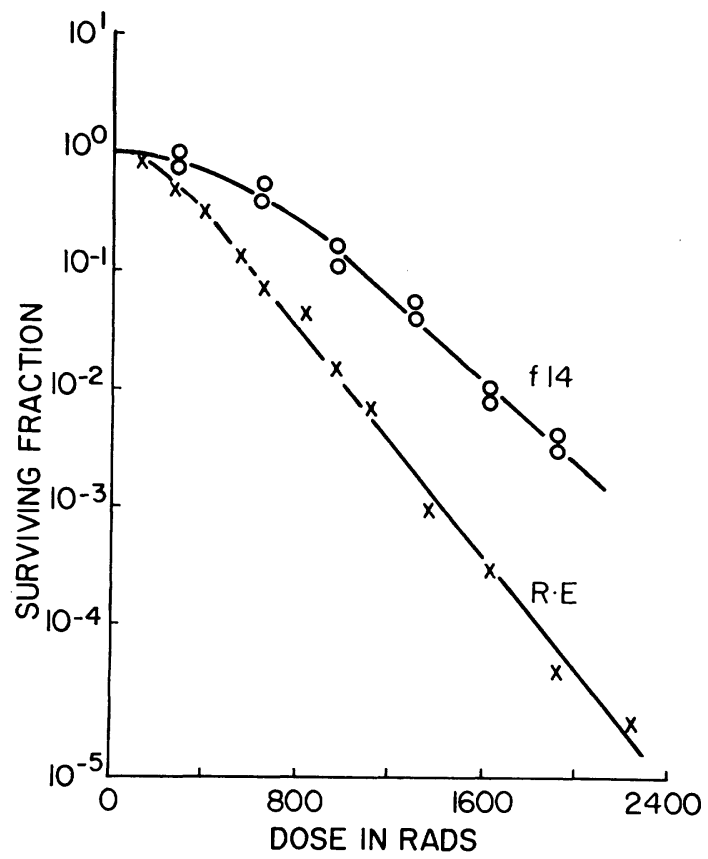

FIg. 1. Radiation survival curves for normal rat embryo cells $(X)$ and cells of the f14 longterm polyoma-transformed focal line $(O)$. Results of two separate experiments. 
lated) rat embryo (R.E.) cells and for a number of transformed focal cell lines were determined. The survival curves obtained in vitro for normal cells and for cells of the 14 long-term transformed line are shown in Fig. 1 . The $D_{0}$ value found for the normal cells, $179 \pm 13 \mathrm{rads}$, is significantly smaller than the $D_{0}$ value found for the f14 line, $252 \pm 37$ rads. Thus, the f14 long-term transformed line showed a radiosensitivity significantly less than that of normal rat embryo cells. The extrapolation numbers obtained from the two survival curves shown in Fig. 1 also differed, with the f14 line yielding the larger (see Table I).

The survival curves obtained for the f12 long-term transformed focal line, assayed both in vitro and in vivo, are given in Fig. 2 . The $D_{0}$ value for cells assayed in vitro, $273 \pm 13$ rads, does not differ significantly from that obtained for the same cells assayed in vivo, $259 \pm 33$ rads. However, both values are again significantly greater than that obtained for normal, noninfected rat embryo cells, $179 \pm 13$ rads (Fig. 1). The extrapolation number for cells assayed in vivo was smaller than the value obtained in vitro.

The survival curve parameters obtained for these cells and for the other cell lines tested are summarized in Table I. In the table, the transformed cell lines are listed in decreasing order of sensitivity. Except for the f8 and f22 lines, which showed $D_{0}$ values similar to that of normal rat embryo cells, the majority of the long-term

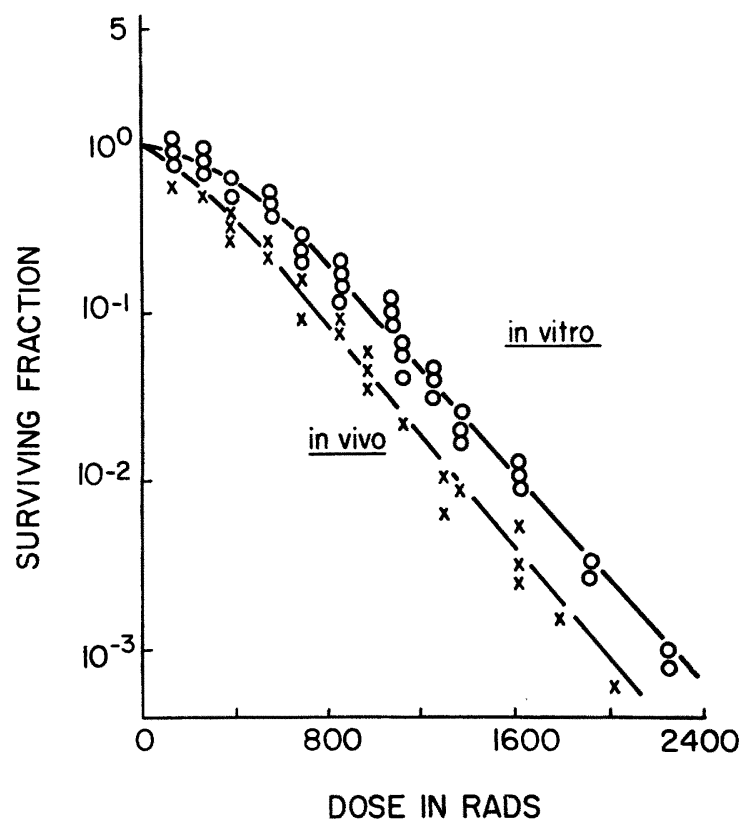

Frg. 2. Radiation survival curves for cells of the f12 long-term transformed line irradiated in vitro and assayed in vitro $(O)$ and in vivo $(X)$. Results of three separate experiments. 
TABLE I

\section{Survival Curve Parameters of Normal Rat \\ Embryo Cells and of Cells of Some \\ Long-Term Transformed Lines}

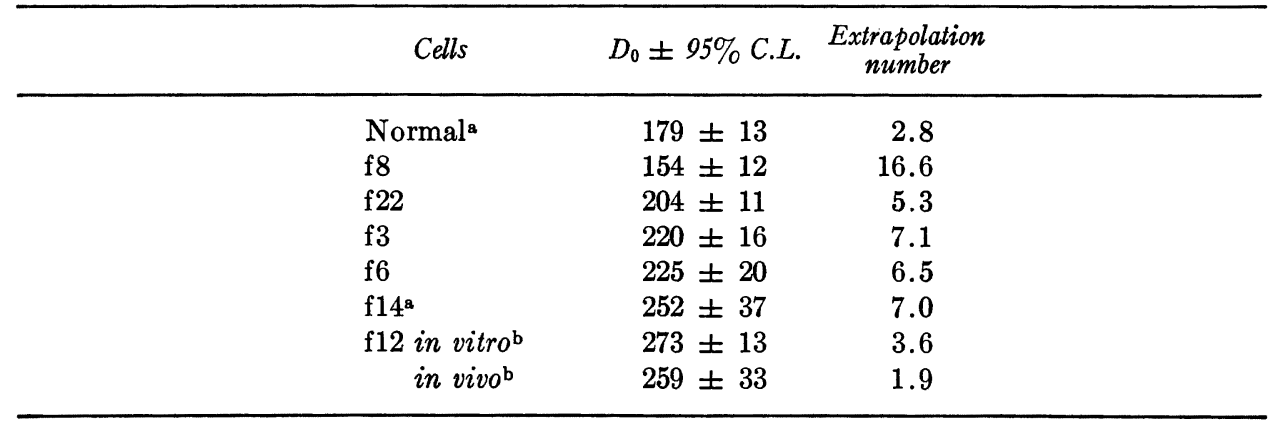

a From Fig. 1.

b From Fig. 2.

transformed cell lines tested yielded $D_{0}$ values significantly greater than that obtained for normal cells. These results indicate that at least some long-term transformed cell lines are much less radiosensitive than normal cells.

Radiosensitivity of Short-Term Transformed Cell Lines and Freshly Transformed Cells

It seemed possible that some long-term transformed cell lines, for instance f12 and $\mathrm{f} 14$, became less radiosensitive as a result of prolonged cultivation in vitro. To test this view, survival curves for short-term transformed cell lines and for newly transformed cells were obtained.

To measure the radiosensitivity of newly transformed cells, two types of experiments were performed. In the first, infected (and sham-infected control) stationaryphase cultures of rat embryo cells were irradiated 36 hours after infection. Suitable numbers of cells were plated, such that discrete colonies were obtained, and differential counts of normal and transformed colonies were made. The results obtained in this experiment are shown in Fig. 3. It may be seen that infected cells giving rise to colonies of either transformed or normal morphology yielded survival data which did not differ significantly from the data obtained for normal, noninfected rat embryo cells.

In the second type of experiment carried out on newly transformed cells, stationary-phase cells were irradiated 7 days after infection and tested for the formation of transformed foci in vitro (8). At the same time, aliquots of the same cell suspension were injected into weanling rats to test for the formation of lung colonies in vivo. The results obtained are depicted in Fig. 4 . The $D_{0}$ value for transformed cells assayed in vitro is $167 \pm 24$ rads, not significantly different from the $D_{0}$ value of 157 rads obtained for cells assayed in vivo, nor from the $D_{0}$ value of $179 \pm 13$ rads 


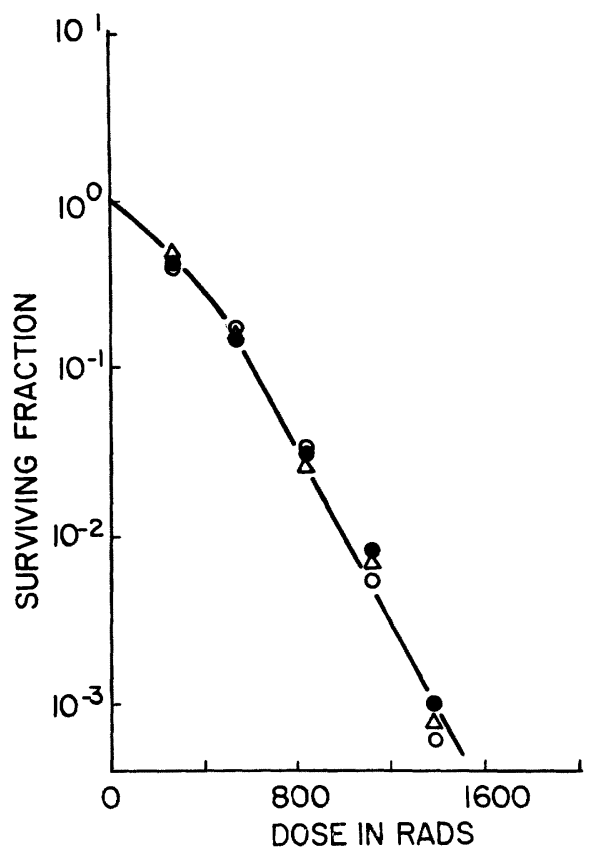

Fig. 3. Survival data for normal and newly transformed cells: $\mathbf{0}$, normal, noninfected cells; $O$, infected cells yielding colonies of normal morphologies; $\triangle$, infected cells yielding colonies of transformed morphologies.

obtained previously for normal, noninfected rat embryo cells (Fig. 1). Again, the extrapolation number was less for the cells assayed in vivo.

These results, together with the results obtained for the cells of three short-term transformed lines, E2, E3, and E4, which had been in culture for not more than 7 weeks, are summarized in Table II. It may be concluded from these results that newly transformed cells and short-term focal cell lines yield $D_{0}$ values very similar to the value obtained for normal cells, and that the $D_{0}$ values for newly transformed cells are the same when assayed in vivo as when assayed in vitro. It seems likely, therefore, that the differences in radiosensitivity observed between normal cells and some of the long-term transformed lines are the result of prolonged cultivation of the latter lines in vitro.

A Comparison of the Radiosensitivity of Various Cell Lines, with Some Properties of These Lines

The prime objective of the present study was to determine if normal and malignant cells differed in cellular radiosensitivity. For this reason, an attempt was made to determine if a consistent relationship existed between radiosensitivity and degree 
of malignancy, as determined by the number of lung colonies formed by a given number of injected cells. In doing so, it must be taken into account that, besides becoming malignant, these transformed cells had acquired other properties as a result of either viral infection or continuous culture in vitro. For instance, the long-

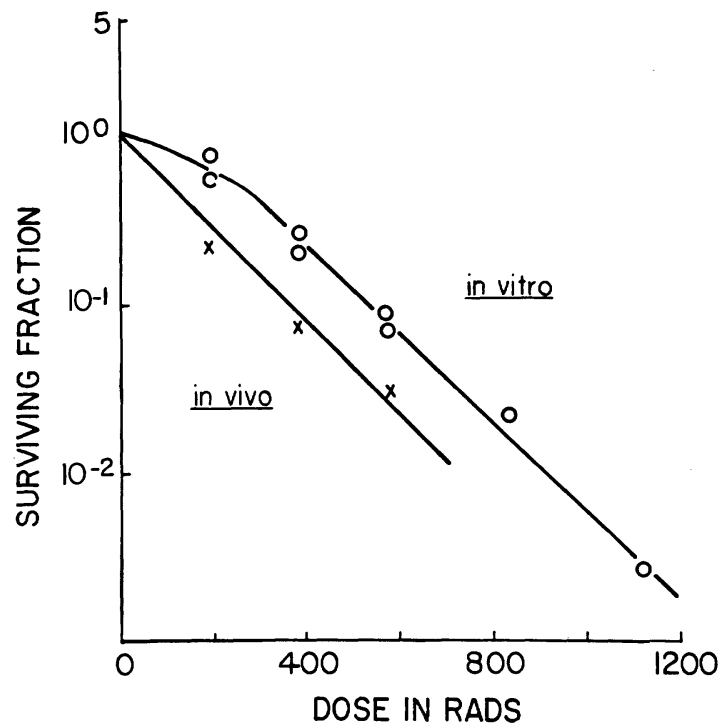

FIg. 4. Radiation survival curves for newly transformed cells irradiated in vitro, and assayed in vitro $(O)$ and in vivo $(X)$. In the unirradiated controls, the transformation frequency per cell in vitro was $2.3 \times 10^{-3}$, and the frequency of formation of lung colonies in vivo per injected cell was $2.7 \times 10^{-6}$.

TABLE II

Survival Curve Parameters of Normal and Freshly

Infected Rat Embryo Cells, and of Some Short-Term Transformed Lines

\begin{tabular}{lcc}
\hline \multicolumn{1}{c}{ Cells } & $D_{0} \pm 95 \%$ C.L. & $\begin{array}{c}\text { Extrapolation } \\
\text { number }\end{array}$ \\
\hline Normal $^{\mathrm{a}}$ & $179 \pm 13$ & 2.8 \\
Infected (36 hours) $^{\mathrm{b}}$ & Same as normal cells \\
Infected (7 days) $^{\mathrm{c}}$ & & \\
$\quad$ In vitro & $167 \pm 24$ & 2.5 \\
In vivo & 157 & 1.0 \\
E3 & $165 \pm 27$ & 5.6 \\
E2 & $182 \pm 14$ & 4.3 \\
E4 & $188 \pm 37$ & 6.3 \\
\hline
\end{tabular}

a From Fig. 1.

b From Fig. 3.

c From Fig. 4. 
TABLE III

Comparison of Radiation Survival Curve Parameters and Parameters of Other Cell Properties

\begin{tabular}{|c|c|c|c|c|c|}
\hline Cells & $\begin{array}{c}D_{0} \text { in vitro } \\
\text { 95\% C.L. }\end{array}$ & $\begin{array}{l}\text { Lung colonies per } \\
\text { injected cell }\end{array}$ & $\begin{array}{c}\text { Doubling } \\
\text { time in } \\
\text { vitro (hours) }\end{array}$ & $\begin{array}{l}\text { Approxi- } \\
\text { mate cell } \\
\text { ploidy }\end{array}$ & $\begin{array}{l}\text { Plating } \\
\text { efficiency in } \\
\text { vitro }(\%)\end{array}$ \\
\hline Normal & $179 \pm 13$ & $<5 \times 10^{-8}$ & 24 & $2 n$ & 16 \\
\hline \multicolumn{6}{|c|}{ Short-term transformed lines } \\
\hline E3 & $165 \pm 27$ & $<1 \times 10^{-6}$ & 21 & $2 n$ & 14 \\
\hline E2 & $182 \pm 14$ & $<1 \times 10^{-6}$ & 24 & $2 n$ & 16 \\
\hline $\mathrm{E} 4$ & $188 \pm 37$ & $<1 \times 10^{-6}$ & 20 & $2 n$ & 21 \\
\hline \multicolumn{6}{|c|}{ Long-term transformed lines } \\
\hline f8 & $154 \pm 12$ & $3.3 \times 10^{-5}$ & 16 & $4 n$ & 22 \\
\hline $\mathrm{f} 22$ & $204 \pm 11$ & $<2 \times 10^{-5}$ & 18 & $2 n$ & 51 \\
\hline f3 & $220 \pm 16$ & $2.5 \times 10^{-2}$ & 15 & $4 n$ & 31 \\
\hline f6 & $225 \pm 20$ & $1.2 \times 10^{-3}$ & 15 & $4 n$ & 26 \\
\hline f14 & $252 \pm 37$ & $1.0 \times 10^{-3}$ & 12 & $4 n$ & 28 \\
\hline $\mathrm{f} 12$ & $273 \pm 13$ & $0.3-4 \times 10^{-2}$ & 16 & $4 n$ & 27 \\
\hline
\end{tabular}

a From Tables I and II.

b Taken from ref. 10 .

term transformed cell lines all had higher plating efficiencies and more rapid growth rates than normal rat embryo cells. In addition, all were tetraploid or hypertetraploid, except the 22 line, which was diploid. Therefore, in attempting to correlate malignancy with radiosensitivity, one must also consider these other properties, since it is possible that they influenced radiosensitivity in some consistent way.

A number of properties were examined for the cells whose radiosensitivity was tested. These were as follows: lung colony-forming efficiency, growth rate in vitro (doubling time of cultures), approximate cell ploidy, and plating efficiency in vitro. The parameters relating to these cell properties are listed in Table III, along with the $D_{0}$ values from the experiments previously discussed. It can be seen that there was no consistent relationship between radiosensitivity (as measured by the $D_{0}$ value) and doubling time, plating efficiency in vitro, or ploidy. There was, however, some correlation between radiosensitivity and degree of malignancy, in terms of lung colony-forming efficiency, though even here the correlation was not good. In general, as can be seen in Table III, the less radiosensitive lines were those with the higher lung colony-forming efficiencies, which were considered to be the most malignant cell lines. The results indicate that at least some of the different tumorforming lines possessed significantly different radiosensitivities.

\section{DISCUSSION}

From the results presented in this paper, it appears that virus-induced transformation does not result in any detectable prompt shift in the radiosensitivity of 
irradiated rat embryo cells. Although some transformed lines were found to exhibit a decreased radiosensitivity in comparison with normal, nontransformed cells, it is probable that this decrease in sensitivity occurred some time after the initial events of transformation. If so, it is likely that the decreased sensitivity either was the secondary result of instability of the transformed cells, or was due to some effect of continued growth of the cells in vitro. It would be of interest from this viewpoint to carry out a study of the variations in radiosensitivity if a given transformed cell line as a function of time in culture after infection, in order to test for the gradual outgrowth of a small number of less sensitive cells.

In the experiments reported here, different lines of transformed cells, isolated in the same way from polyoma-infected rat embryo cultures, and tested by the same procedures both in vitro and in vivo, were found to exhibit different radiosensitivities of colony-forming ability. This finding does not support the view which has arisen out of the work of Morkovin and Feldman (20), and Hewitt (21), that mammalian cells may possess a characteristic, uniform radiosensitivity independent of cellular origin. Instead, it seems likely that the differences observed between different lines of cells, tested by various techniques in a number of laboratories (1), are genuine.

Attempts to correlate the different radiosensitivities observed for the different transformed cells with differences in other cell properties were relatively unsuccessful. The only reasonably consistent correlation was that found between the $D_{0}$ values and the degree of malignancy of the cells, as determined by their efficiencies of formation of lung colonies in vivo (Table III). In general, the cells with the lowest efficiency of formation of lung colonies showed the greatest radiosensitivity. However, in view of the relatively small number of cell lines tested in the present study, further quantitative evidence for such a correlation must be obtained before it may be considered as established.

With the polyoma-transformed rat embryo cell system it was possible to irradiate a suspension of transformed cells in vitro and test for the survival of colony-forming ability of the irradiated cells both in vitro and in vivo. It was found that the survival parameter, $D_{0}$, for the transformed cells was independent of the assay conditions used despite the wide difference in colony-forming efficiencies observed for the same cells in vitro and in vivo, and despite the fact that many more cell divisions are required to form a macroscopic colony in vivo than in vitro. These results provide further support (22, 23) for the view that the $D_{0}$ values measured for cells in vitro are indicative of the $D_{0}$ values for cells in vivo. It was also found that the extrapolation numbers of the survival curves obtained in vivo were smaller than the extrapolation numbers of the survival curves obtained in vitro. The significance of this observation remains to be determined.

It should be noted that the proportion of the cell population which was observed to form colonies was very different in vitro and in vivo. The plating efficiencies in vitro for the cell lines used in this study were 15: or better, while the efficiencies of formation of lung colonies in vivo by these same lines did not exceed $4 \%$, and were 
usually much lower (Table IV). The small subpopulation which was capable of forming lung colonies in vivo may not have been part of the subpopulation capable of colony-formation in vitro, nor were either of these subpopulations necessarily representative of the cell population as a whole. For this reason, the observation that the $D_{0}$ values were similar for the two subpopulations does not provide conclusive proof that the total cell population responds similarly to irradiation in vivo and in vitro. Such proof could be obtained only if conditions could be found such that all the cells were capable of colony formation both in vitro and in vivo. In spite of this limitation on the present system, the studies described in this paper indicate that the transformed rat embryo cell system provides a useful means for the investigation of the effects of ionizing radiations, or of other cytotoxic agents, on the proliferation of normal and malignant cells, both in vitro and in vivo.

\section{SUMMARY}

1. Radiation survival curves have been obtained for freshly isolated normal rat embryo cells, and for transformed cell lines derived from polyoma-infected cultures and tested for their response to irradiation after short (less than 7 weeks) or long (at least 35 weeks) periods of cultivation in vitro.

2. It was found that newly transformed cells and transformed cell lines which had been cultivated in vitro for less than 7 weeks yielded survival curves characterized by $D_{0}$ values which did not differ significantly from the $D_{0}$ value obtained for normal, noninfected cells. In contrast to this, the survival curves obtained for cells which had been cultivated in vitro for more than 35 weeks yielded results which indicated that the radiosensitivities of at least some of these cell lines, as measured by their $D_{0}$ values, were significantly different from each other, and significantly less than the radiosensitivity of normal cells.

3. The survival curves for cells irradiated in vitro and assayed in vitro yielded $D_{0}$ values which were similar to those obtained for the same cells irradiated in vitro but assayed in vivo.

4. A comparison of the $D_{0}$ values obtained for different lines of transformed cells with other properties of the cells, including efficiency of formation of colonies in vitro and in vivo, growth rate, and ploidy, yielded no consistent correlations, with the possible exception of the efficiency of formation of lung colonies in vivo.

\section{ACKNOWLEDGMENTS}

This work was supported in part by grants from the National Cancer Institute of Canada, and the Banting Research Foundation.

ReCeived: February 21, 1966

\section{REFERENCES}

1. G. F. Whitmore and J. E. Till, Quantitation of cellular radiobiological responses. Ann. Rev. Nucl. Sci. 14, 347-374 (1964).

2. T. T. Puck and P. I. Marcus, Action of X-rays on single mammalian cells. J. Exptl. Med. 103, 653-666 (1956). 
3. T. T. Puck, D. Morkovin, P. I. Marcus, and S. J. Cieciura, Action of X-rays on mammalian cells. II. Survival curves of cells from normal human tissues. J. Exptl. Med. 106, 485-500 (1957).

4. H. B. Hewitt and C. W. Wilson, A survival curve for mammalian leukemia cells irradiated in vivo (implications for the treatment of mouse leukemia by whole-body irradiation). Brit. J. Cancer 13, 69-75 (1959).

5. J. E. Till and E. A. MCCulloch, A direct measurement of the radiation sensitivity of normal mouse bone marrow cells. Radiation Res. 14, 213-222 (1961).

6. M. Vogt and R. Dulbecco, Virus-cell interaction with a tumor-producing virus. Proc. Natl. Acad. Sci. U.S. 46, 365-370 (1960).

7. L. SACHS and D. MEdina, In vitro transformation of normal cells by polyoma virus. Nature 189, 457-458 (1961).

8. J. F. Williams and J. E. Till, Transformation of rat embryo cells in culture by polyoma virus. Virology 24, 505-508 (1964).

9. J. F. Williams and J. E. Till, Formation of tumor colonies in the lungs of rats injected with rat embryo cells immediately after infection of the cells with polyoma virus. Virology 27, 625-630 (1965).

10. J. F. Williams and J. E. Till, Formation of lung colonies by polyoma-transformed rat embryo cells. J. Natl. Cancer Inst. in press.

11. R. Dulbecco and M. Vogt, Plaque-formation and isolation of pure lines with poliomyelitis virus. J. Exptl. Med. 99, 167-182 (1954).

12. R. C. Parker, Methods of Tissue Culture, 3rd ed., Hoeber-Harper, New York, 1961.

13. C. P. Stanners, Studies on the transformation of hamster embryo cells in culture by polyoma virus II. Selective techniques for the detection of transformed cells. Virology 21, 464-476 (1963).

14. J. R. Cunningham, W. R. Bruce, and H. P. Webb, A convenient ${ }^{137} \mathrm{Cs}$ unit for irradiating cell suspensions and small laboratory animals. Phys. Med. Biol. 10, 381-384 (1965).

15. Physical Aspects of Irradiation, Recommendations of the International Commission on Radiological Units and Measurements. Natl. Bur. Std. (U.S.) Handbook 85 (1964).

16. T. T. Puck, P. I. Marcus, and S. J. Cieciura, Clonal growth of mammalian cells in vitro, Growth characteristics of colonies from single HeLa cells with and without a "feeder" layer. J. Exptl. Med. 103, 273-284 (1956).

17. M. Stoker and I. MacPherson, Studies on the transformation of hamster cell by polyoma virus in vitro. Virology 14, 359-370 (1961).

18. T. Alper, N. E. Gillies, and M. M. Elkind, The sigmoid survival curve in radiobiology. Nature 186, 1062-1063 (1960).

19. M. Fox and I. M. Zeiss, Chromosome preparation from fresh and cultured tissues using a modification of the drying technique. Nature 192, 1213-1214 (1961).

20. D. Morkovin and A. Feldman, End point of one of the actions of radiations on living tissue important in radiation therapy and in acute radiation syndrome. Brit. J. Radiol. 33, 197 (1960).

21. H. B. HewitT, Fundamental aspects of the radiotherapy of cancer, The Scientific Basis of Medicine Annual Reviews, pp. 305-326, Athlone Press, London, 1962.

22. G. W. Philpott, C. W. Schaeffer, Jr. and L. J. Tolmach, Reproductive survival of chick embryo cells irradiated in ovo and in vitro. Radiation Res. 17, 508-513 (1962).

23. K. B. Dawson, E. O. Field, and H. Madoc-Jones, A comparison of the in vitro and in vivo radiosensitivities of a solid tumour in rats. (Abstract). Intern. J. Radiation Biol. 8, 392 (1964). 